\title{
Management of Acute Type A Aortic Dissection at a Public Cardiac Center in the Northeast Region of Brazil
}

Pablo Cesar Lustosa Barros Bezerra ${ }^{1,2,3}$, MD; Ricardo de Carvalho Lima ${ }^{1,3}$, MD, MSc, PhD; Pedro Rafael de Salerno ${ }^{1,3}$, MD, MSc, PhD; Antonio Cavalcanti de Albuquerque Martins², MD, MSc, PhD; Geovanna Menezes de Medeiros Lustosa ${ }^{2}, \mathrm{MSc}_{\text {; Alvaro Monteiro Perazzo }}^{4}$, MD; Juliana Vieira de Oliveira Salerno ${ }^{5}$, MD; Carolina Vieira de Oliveira Salerno ${ }^{6}$, BSc; Pedro Rafael Vieira de Oliveira Salerno ${ }^{7}$, BSc

DOI: $10.21470 / 1678-9741-2020-0169$

\section{Abstract}

Introduction: Aortic diseases are among the most serious cardiovascular diseases; the overall mortality rate due to diseases such as aneurysms and aortic dissections has been estimated at 2.78 per 100,000 persons in 2010 , with a higher mortality rate in men than women. Our objective was to evaluate the epidemiological profile of patients with acute type $A$ aortic dissection at a cardiology referral center.

Methods: A retrospective cross-sectional study was performed at a public cardiac center with 24 patients hospitalized from $1 / 1 / 2016$ to $12 / 31 / 2017$ with a confirmed diagnosis of acute type A aortic dissection.

Results: Twenty (83.3\%) out of 24 patients underwent surgery and four $(16.7 \%)$ did not undergo surgery. Among those who underwent surgery, $10(50 \%)$ died and $10(50 \%)$ were discharged, and all non-operated patients died $(P=0.114)$ (Fisher's exact test).
The male gender predominated $(n=19,79.2 \%), 86.7 \%(n=13)$ of the patients presented body mass index $>25 \mathrm{~kg} / \mathrm{m}^{2}$, chest pain was found in $91.7 \%(n=22)$, and renal failure was present in $45.8 \%$ $(n=11)$ of the cases. Hypertension predominated in $91.7 \%(n=22)$ and the main exam was aortic angiotomography in $79.2 \%(n=19)$ of the cases.

Conclusion: The study presented a small sample size, making it impossible to associate the factors, although the service was considered a high-volume referral center. It is possible that the delay in arriving at the service and the accomplishment of invasive imaging with the use of contrast agents have aggravated the patients' condition and have been decisive for the increase in lethality, which requires further studies.

Keywords: Aneurysm, Dissecting. Aorta. Hypertension. Aortic Diseases. Cardiovascular Diseases. Body Mass Index. Referral and Consultation.

\begin{tabular}{llll}
\hline Abbreviations, acronyms \& symbols & & \\
\hline AAS & = Acute aortic syndromes & MACCE & $=$ Major adverse cardiovascular and cerebrovascular events \\
AD $\quad$ = Aortic dissection & MRI & $=$ Magnetic resonance imaging \\
BMI $\quad=$ Body mass index & PAU & $=$ Penetrating atherosclerotic ulcer \\
CT $\quad=$ Computed tomography & PROCAPE = Pronto-Socorro Cardiológico de Pernambuco \\
ICU $\quad=$ Intensive care unit & SD & $=$ Standard deviation \\
IMH $\quad=$ Intramural haematoma & TEE & $=$ Transesophageal echocardiogram \\
IRAD $=$ International Registry of Acute Aortic Dissection & TTE & $=$ Transthoracic echocardiogram
\end{tabular}

'Division of Cardiovascular Surgery, Pronto-Socorro Cardiológico de Pernambuco PROCAPE, Universidade de Pernambuco - UPE, Recife, Pernambuco, Brazil.

${ }^{2}$ Postgraduation Department, Instituto de Medicina Integral Professor Fernando Figueira, Recife, Pernambuco, Brazil.

${ }^{3}$ Department of Cardiovascular Surgery, Universidade de Pernambuco - UPE, Recife, Pernambuco, Brazil.

${ }^{4}$ Department of Cardiovascular Surgery, Universidade de Pernambuco - UPE, Recife, Pernambuco, Brazil.

${ }^{5}$ Department of in General Surgery, Hospital da Restauração - SES-PE, Recife, Pernambuco, Brazil.

6Department of Universidade Federal de Pernambuco - UFPE, Recife, Pernambuco, Brazil. 7Department of Faculdade Pernambucana de Saúde - FPS, Recife, Pernambuco, Brazil.
This study was carried out at the Division of Cardiovascular Surgery, Pronto-Socorro Cardiológico de Pernambuco - PROCAPE, Universidade de Pernambuco - UPE, Recife, Pernambuco, Brazil.

Correspondence Address: Pablo Cesar Lustosa Barros iD https://orcid.org/0000-0003-3029-791X

Division of Cardiovascular Surgery, Pronto-Socorro Cardiológico de Pernambuco PROCAPE, Universidade de Pernambuco - UPE

Rua dos Palmares, S/N, Santo Amaro, Recife, PE, Brazil - Zip Code: 50100-010 E-mail: pablolustosa@yahoo.com.br, pablolustosa@gmail.com 


\section{INTRODUCTION}

Cardiovascular diseases are leading causes of death, accounting for $29.8 \%$ of all causes of death in Brazil in $2013^{[1]}$ and 31.3\% of them in the world in 2015 (World Health Organization - WHO). Among all cardiovascular diseases, aortic diseases, divided into aneurysms, dissections, congenital diseases, and trauma, stand out ${ }^{[2,3]}$.

Acute aortic syndromes (AAS) are defined as emergency conditions with similar clinical characteristics involving the aorta. Physiopathologically, the origin is common to the various types of AAS that eventually start with rupture of the intima and middle layers of the aortic wall, which can result in intramural haematoma $(\mathrm{IMH})$, penetrating atherosclerotic ulcer (PAU), or even in the separation of the layers of the aortic wall, causing aortic dissection (AD) or even complete aortic rupture ${ }^{[3]}$.

AAS occurs when a small lesion or ulcer allows blood to penetrate through the aortic lumen towards its middle layer or when the vasa vasorum ruptures, causing bleeding in the middle layer. The inflammatory response to blood in the middle layer can lead to aortic dilation and rupture ${ }^{[3]}$.

ADs are classified according to the time of symptom onset and type of symptom (according to the site of involvement). Regarding the time of symptom onset, they are usually classified as acute (up to 14 days), subacute (15-90 days), and chronic (> 90 days ${ }^{[3]}$. Regarding the site of involvement, they are classified as Stanford ${ }^{[4-9]}$ type $A$ (with involvement of the ascending aorta) and type $B$ (without involvement of the ascending aorta) ${ }^{[3-5]}$.

$A D s$ are believed to begin with the formation of a tear in the aortic intima that directly exposes an underlying diseased middle layer to the driving force of intraluminal blood, separating the layers of the aortic wall and the subsequent formation of a false lumen, with or without communication. In most cases, a small lesion of the intima is the starting condition, causing blood to travel through a dissection plane through the middle layer. This process causes both the rupture of the aorta, in the case of rupture of the adventitial layer, and the reentry into the true lumen through a second failure in the intimal layer ${ }^{[3,6,7]}$.
The dissection can be antegrade or retrograde. Complications include tamponade, aortic valve insufficiency, and proximal or distal malperfusion syndrome $e^{[3,6,7]}$. The inflammatory response to thrombosis of the middle layer is susceptible to initiate additional necrosis and apoptosis of smooth muscle cells, in addition to the degeneration of elastic tissue, factors that increase the risk of rupture of the middle layer ${ }^{[3]}$.

Currently, epidemiological data on AD are scarce. The incidence of $A D$ is estimated at six cases for every 100,000 persons per year, with a higher mortality rate in men than in women and increasing with age $\mathrm{e}^{[3]}$. The prognosis is worse in women, as a consequence of an atypical presentation of the disease and delayed diagnosis. The main risk factor associated with $A D$ is hypertension, observed in $65-75 \%$ of the individuals, which is commonly poorly controlled ${ }^{[3,6-8]}$. In The International Registry of Acute Aortic Dissection (IRAD), the patients' mean age was 63 years and $65 \%$ of them were men. Other associated risk factors are: pre-existing aortic disease or aortic valve disease, family history of aortic diseases, history of cardiac surgery, smoking, direct trauma to the chest, and use of intravenous drugs (e.g." cocaine and amphetamines ${ }^{[3,6,7]}$.

Acute type A AD frequently presents with chest pain (in $80 \%$ of cases), with a sudden onset, but it can present as back pain or migratory pain. AD can complicate with aortic valve failure (40$75 \%$ of cases), cardiac tamponade (20\% of cases), renal failure (20\% of cases), myocardial ischemia or infarction (10-15\%), and even coma or stroke (10\% of cases $)^{[3,6,7]}$.

The main objective of imaging studies in acute AD is the comprehensive assessment of the entire aorta, including its diameter, shape, and extent of dissection, involvement of the aortic valve, branches of the aorta, relationship with adjacent structures, and the presence of intramural thrombus ${ }^{[3]}$.

The main imaging tests used in the diagnosis of $A D$ are transthoracic echocardiogram (TTE), transesophageal echocardiogram (TEE), computed tomography (CT), magnetic resonance imaging (MRI), and aortography. However, CT and MRI have been considered superior to TEE and TTE to assess the extension and involvement of branches in cases of $A D$, as well
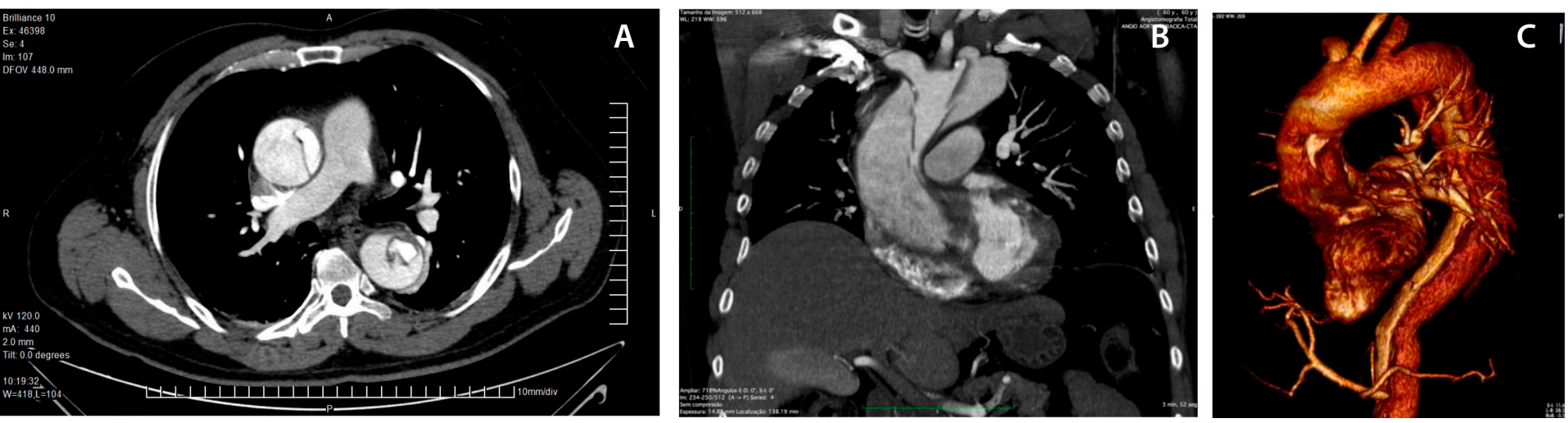

Fig. 1A to C - Angiotomography of one patient diagnosed with type A acute aortic dissection: A) transversal view; B) frontal view; C) 3D reconstruction view. 
as for the diagnosis of $\mathrm{IMH}, \mathrm{PAU}$, and traumatic aortic injuries ${ }^{[9]}$. Figures $1 \mathrm{~A}$ to $1 \mathrm{C}$ show three different views of the aorta from a CT angiotomography.

Drug therapy for pain control and hemodynamic control is essential. In acute type A AD, surgery is the treatment of choice ${ }^{[3]}$.

Acute type $A$ AD has a $50 \%$ mortality rate in the first 48 hours if not operated ${ }^{[3,5]}$. Despite improvements in surgical and anesthetic techniques, perioperative mortality (25\%) and neurological complications (18\%) remain high ${ }^{[3,9,10]}$. However, surgery reduces mortality in one month from $90 \%$ to $30 \%{ }^{[3,7,9]}$. The advantage of surgery over conservative treatment is particularly obvious in long-term follow-up ${ }^{[5,9]}$. Based on this evidence, all patients with acute type A AD should be referred to surgery; however, coma, shock secondary to pericardial tamponade, poor coronary or peripheral artery perfusion, and stroke are important predictive factors for postoperative mortality ${ }^{[3,9,10]}$.

As mentioned, acute type A AD is a disease with a high lethality rate, little is known about the epidemiology of type $A$ AD in Brazil and in its North and Northeast regions, especially in the state of Pernambuco, as well as its impact on patient survival. Therefore, the aim of this study is to evaluate the epidemiology of patients with acute type A AD treated at the Pronto-Socorro Cardiológico de Pernambuco (PROCAPE) and to analyze the results obtained at the institution, so that it is possible to list proposals for improvements in local health care for this highly lethal condition.

\section{METHODS \\ Study Design}

The study was carried out at a public cardiac center in the Northeast of Brazil, being of a descriptive, retrospective crosssectional nature, to assess the lethality and epidemiological profile of patients with acute type A AD, after confirmation of their diagnosis. Data collection was performed through electronic medical records, using as a sample the entire population of patients diagnosed with acute type A AD admitted to PROCAPE between 01/01/2016 and 12/31/2017.

Inclusion criteria were: patients diagnosed with AAS classified within ICD10-71 and who were hospitalized in that period. Exclusion criteria were: patients admitted and not diagnosed with acute type A AD during hospitalization.

The selected cases were included in a database spreadsheet previously coded in Microsoft Excel format with all the variables described by the researcher and contemplated by the patient in question, for further data analysis.

Two persons typed the data, at different times, making it possible to compare the two databases and identify possible typing errors, through consistency and cleaning tests. Only after completing these steps, the definitive database was used for statistical analysis.

The researcher analyzed the data using IBM Corp. Released 2011, IBM SPSS Statistics for Windows, Version 20, Armonk, NY: IBM Corp (or a newer version of it), with which were performed the calculation of the mortality rate of patients who underwent surgery and the calculation of the lethality rate of patients who did not undergo surgery. It was not possible to perform the logistic regression of the possible associated factors, as well as the calculation of average times and standard deviation between the procedures that patients went through during hospitalization, due to the low total number of cases found in this study, making it impossible to establish a relationship between outcomes and associated factors. No correlation analysis of any type of data was performed, nor any association between risk factors and death, due to the low total number of cases found in this study. The outcome tables were cross-checked with the surgery variable, and Fisher's exact test was used for analysis. In all stages of the analysis, the significance level of 5\% was considered, adopting two-tailed P-values. Due to the factors mentioned above, multivariate analysis was dispensed with.

\section{Ethical Aspects}

The study respected the ethical principles of the Declaration of Helsinki, resolution 466/2012, and was submitted to the Research Ethics Committee of the University of Pernambuco - UPE, under CAAE 88050618.2.0000.5192, approved on $06 / 14 / 2018$. A waiver of informed consent was requested. Data confidentiality was guaranteed.

\section{RESULTS}

In the period from 01/01/2016 to $12 / 31 / 2017,100$ patients with a diagnosis of aortic syndromes were admitted, of which 76 were excluded (62 for fulfilling the exclusion criteria and 14 for error in the electronic medical record) and 24 was the total of confirmed cases of acute type A AD.

Parametric variables (Table 1) did not differ between the groups, a significant relationship was found only between hypertension and surgical exposure, showing that among the cases that were operated on, 100\% had hypertension, against $50 \%$ of those that were not operated on $(P=0.022)$, although without clinical significance.

Non-parametric variables (Table 2) did not differ in most cases. An average body mass index (BMI) of $28.63 \mathrm{~kg} / \mathrm{m}^{2}$ with a standard deviation of $3.00 \mathrm{~kg} / \mathrm{m}^{2}$ among patients who died and an average BMI of $33.42 \mathrm{~kg} / \mathrm{m}^{2}$ with a standard deviation of $5.6 \mathrm{~kg} / \mathrm{m}^{2}$ among patients discharged were found ( $P=0.045$ ). An average intensive care unit (ICU) length of stay after surgery of 3.32 days with a standard deviation of 4.45 days among patients who died and 5.75 days with a standard deviation of 3.13 days among patients who were discharged were observed $(P=0.039)$. An average length of hospital stay of 5.81 days with a standard deviation of 7.56 days among patients who died and 26.97 days with a standard deviation of 20.45 days among those who were discharged from hospital were also found $(P=0.001)$. A significant relationship was found only with the total length of hospital stay, with an average of 17.31 days and standard deviation of 18.14 days among patients who underwent surgery and 1.23 days and standard deviation of 1.22 days among those who did not undergo surgery $(P=0.007)$.

Between the final outcome and the surgical intervention, and as an exposure factor and main modifier of prognosis (Figura 2), it was shown that $100 \%$ of the patients who did not undergo surgery died during hospitalization and, of those who had 
Table 1. Results of parametric variables (social, biological, clinical, surgical, and ICU).

\begin{tabular}{|c|c|c|c|c|c|c|c|c|c|c|c|c|}
\hline \multirow{3}{*}{$\begin{array}{l}\text { Biological, social, clinical, and surgical } \\
\text { variables }\end{array}$} & \multicolumn{2}{|c|}{ Total } & \multicolumn{5}{|c|}{ Final outcome } & \multicolumn{5}{|c|}{ Exposure } \\
\hline & \multirow[t]{2}{*}{$\mathrm{n}$} & \multirow[t]{2}{*}{$\%$} & \multicolumn{2}{|c|}{ Death } & \multicolumn{2}{|c|}{ Discharge } & \multirow{2}{*}{$P$-value* } & \multicolumn{2}{|c|}{ Operated } & \multicolumn{2}{|c|}{ Non-operated } & \multirow{2}{*}{$P$-value* } \\
\hline & & & $n$ & $\%$ & $n$ & $\%$ & & $n$ & $\%$ & $\mathrm{n}$ & $\%$ & \\
\hline Gender & & & & & & & 0.358 & & & & & 0.554 \\
\hline Male $(n, \%)$ & 19 & 79.2 & 10 & 71.4 & 9 & 90.0 & & 15 & 75.0 & 4 & 100.0 & \\
\hline Female (n, \%) & 5 & 20.8 & 4 & 28.6 & 1 & 10.0 & & 5 & 25.0 & 0 & 0 & \\
\hline Proceedings & & & & & & & 1.000 & & & & & 0.136 \\
\hline Metropolitan Region of Recife & 18 & 75.0 & 11 & 78.6 & 7 & 70.0 & & 16 & 80.0 & 2 & 50.0 & \\
\hline Other cities & 4 & 16.7 & 2 & 14.3 & 2 & 20.0 & & 2 & 10.0 & 2 & 50.0 & \\
\hline Other states & 2 & 8.3 & 1 & 7.1 & 1 & 10.0 & & 2 & 10.0 & 0 & 0 & \\
\hline \multicolumn{13}{|l|}{ Symptoms on admission ${ }^{\text {a }}$} \\
\hline Chest pain & 22 & 91.7 & 12 & 85.7 & 10 & 100.0 & 0.493 & 18 & 90.0 & 4 & 100.0 & 1.000 \\
\hline Dyspnea & 4 & 16.7 & 4 & 28.6 & - & - & 0.114 & 2 & 10.0 & 2 & 50.0 & 0.115 \\
\hline Syncope & 3 & 12.5 & 2 & 14.3 & 1 & 10.0 & 1.000 & 3 & 15.0 & 0 & 0 & 1.000 \\
\hline \multicolumn{13}{|l|}{ Dissection complications $^{b}$} \\
\hline Cardiac tamponade & 2 & 8.3 & 2 & 14.3 & - & - & 0.493 & 1 & 5.0 & 1 & 25.0 & 0.312 \\
\hline Mesenteric ischemia & 1 & 4.2 & 1 & 7.1 & 0 & 0.0 & 1.000 & 1 & 5.0 & 0 & 0.0 & 1.000 \\
\hline Major neurologic deficit (MACCE) & 1 & 4.2 & 0 & 0.0 & 1 & 10.0 & 0.417 & 1 & 5.0 & 0 & 0.0 & 1.000 \\
\hline Limb ischemia & 3 & 12.5 & 2 & 14.3 & 1 & 10.0 & 1.000 & 3 & 15.0 & 0 & 0.0 & 1.000 \\
\hline Renal failure & 11 & 45.8 & 5 & 35.7 & 6 & 60.0 & 0.408 & 9 & 45.0 & 2 & 50.0 & 1.000 \\
\hline $\begin{array}{l}\text { Myocardial ischemia or acute myocardial } \\
\text { infarction }\end{array}$ & 5 & 20.8 & 3 & 21.4 & 2 & 20.0 & 1.000 & 5 & 25.0 & 0 & 0.0 & 0.544 \\
\hline \multicolumn{13}{|l|}{ Comorbidities $^{c}$} \\
\hline Hypertension & 22 & 91.7 & 12 & 85.7 & 10 & 100.0 & 0.493 & 20 & 100.0 & 2 & 50.0 & 0.022 \\
\hline Diabetes mellitus & 3 & 13.0 & 1 & 7.1 & 2 & 20.0 & 0.550 & 3 & 15.0 & 0 & 0.0 & 1.000 \\
\hline Smoking & 5 & 20.8 & 4 & 28.6 & 1 & 10.0 & 0.358 & 3 & 15.0 & 2 & 50.0 & 0.179 \\
\hline \multicolumn{13}{|l|}{ Medication in use } \\
\hline Clopidogrel & 2 & 8.3 & 1 & 7.1 & 1 & 10.0 & 1.000 & 2 & 10.0 & 0 & 0.0 & 1.000 \\
\hline Confirmatory diagnostic tests & & & & & & & 0.059 & & & & & 1.000 \\
\hline Transthoracic echocardiogram & 3 & 12.5 & 0 & 0.0 & 3 & 30.0 & & 3 & 15.0 & 0 & 0.0 & \\
\hline Aortic angiotomography & 19 & 79.2 & 13 & 92.9 & 6 & 60.0 & & 15 & 75.0 & 4 & 100.0 & \\
\hline Aortography & 2 & 8.3 & 1 & 7.1 & 1 & 10.0 & & 2 & 10.0 & 0 & 0.0 & \\
\hline Diagnosis confirmed before admission & 7 & 30.4 & 2 & 14.3 & 5 & 55.6 & 0.66 & 6 & 31.6 & 1 & 25.0 & 1.000 \\
\hline \multicolumn{13}{|l|}{ ICU variables } \\
\hline ICU admission before surgery & 16 & 80.0 & 11 & 78.6 & 7 & 70.0 & 0.665 & 16 & 80.0 & 2 & 50.0 & 0.251 \\
\hline Discharge from the ICU before surgery & 2 & 10.0 & 1 & 7.1 & 1 & 10.0 & 1.000 & 2 & 10.0 & 0 & 0.0 & 1.000 \\
\hline Admission to the ICU after surgery & & & 6 & 60.0 & 10 & 100.0 & 0.087 & & & & & \\
\hline Discharge from the ICU after surgery & & & 0 & 0.0 & 10 & 100.0 & 0.000 & & & & & \\
\hline Non-operated patients admitted to the ICU & 2 & 50.0 & & & & & & & & & & \\
\hline \multicolumn{13}{|l|}{ Surgical variables } \\
\hline Reoperation & 4 & 20.0 & 2 & 20.0 & 2 & 20.0 & 1.000 & & & & & \\
\hline $\begin{array}{l}\text { Number of type A AD surgeries in service } \\
\text { per year (mean } \pm \text { SD) }\end{array}$ & 10 & \pm 3 & & & & & & & & & & \\
\hline
\end{tabular}

$\mathrm{a}, \mathrm{b}, \mathrm{c}$ There may be more than one symptom, complication, and comorbidity in each case

*Fisher's exact test

$\mathrm{AD}=$ aortic dissection; $\mathrm{ICU}=$ =intensive care unit; $\mathrm{MACCE}=$ major adverse cardiovascular and cerebrovascular events; $\mathrm{SD}=$ standard deviation 


\begin{tabular}{|c|c|c|c|c|c|c|c|c|c|c|c|c|c|c|c|c|c|c|c|c|c|c|c|c|}
\hline \multirow{5}{*}{ 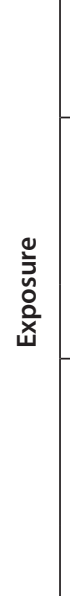 } & \multicolumn{2}{|c|}{ 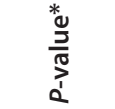 } & $\mid \begin{array}{c}\tilde{\sigma} \\
\tilde{\tilde{\sigma}} \\
0\end{array}$ & \begin{tabular}{|c}
$\bar{\infty}$ \\
0 \\
0
\end{tabular} & $\mid \begin{array}{l} \pm \\
\infty \\
0 \\
0\end{array}$ & $\begin{array}{l}\text { 岕 } \\
\infty \\
0 \\
0\end{array}$ & & & \begin{tabular}{|l} 
\& \\
\multirow{2}{0}{} \\
0
\end{tabular} & $\begin{array}{l}\text { त̂ } \\
\text { Oें }\end{array}$ & & ' & & & & & ' & & ' & & $\frac{\infty}{\frac{\infty}{0}}$ & & $\begin{array}{l}\hat{0} \\
0\end{array}$ & \\
\hline & \multirow{2}{*}{ 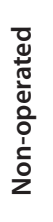 } & 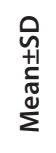 & 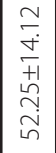 & $\frac{\substack{n \\
m}}{\dot{m}}$ & 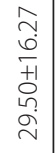 & $\begin{array}{l}\text { ô } \\
\stackrel{1}{1} \\
+1 \\
\infty \\
\stackrel{0}{-}\end{array}$ & & & 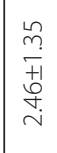 & 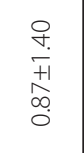 & & ' & & 1 & $\begin{array}{l}\text { त̂ } \\
\text { + } \\
\stackrel{1}{1} \\
\text { N }\end{array}$ & & 1 & 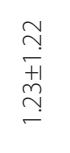 & ' & & 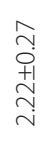 & 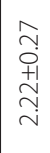 & $\begin{array}{l}\underset{N}{\sim} \\
\underset{\sim}{+1} \\
\stackrel{\sim}{\simeq}\end{array}$ & \\
\hline & & $=$ & $\nabla$ & - & 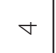 & $m$ & & ' & t & $m$ & & , & & & $\sim$ & ' & 1 & $\nabla$ & , & & $\sim$ & $\sim$ & $\nabla$ & \\
\hline & \multirow{2}{*}{ 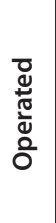 } & 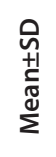 & 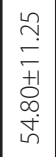 & $\begin{array}{l}\stackrel{9}{q} \\
\omega \\
+1 \\
o \\
+ \\
\dot{m} \\
\end{array}$ & 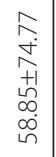 & 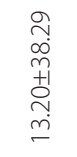 & & ' & 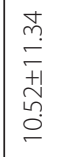 & $\begin{array}{l}\text { fे } \\
\text { +े } \\
+1 \\
\text { \&े } \\
\text { in }\end{array}$ & 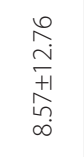 & 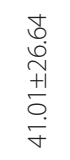 & 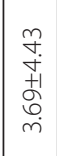 & $\begin{array}{l}0 \\
\infty \\
\stackrel{\infty}{I} \\
\stackrel{+1}{+1} \\
\infty \\
\infty \\
\infty \\
-\end{array}$ & & 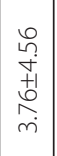 & 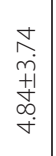 & & $\begin{array}{l}\bar{m} \\
\infty \\
+1 \\
\stackrel{d}{\wedge} \\
\wedge\end{array}$ & 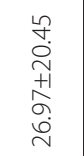 & $\begin{array}{l}8 \\
0 \\
+1 \\
+1 \\
\infty \\
0 \\
0\end{array}$ & & 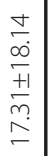 & \\
\hline & & $=$ & থ & \pm & $\stackrel{\sim}{*}$ & $\simeq$ & & ' & 은 & $a$ & $\underline{m}$ & 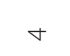 & 0 & 으 & & 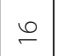 & $\stackrel{\bullet}{-}$ & , & $\therefore$ & $\circ$ & i & & $\stackrel{\sim}{~}$ & \\
\hline \multirow{5}{*}{ 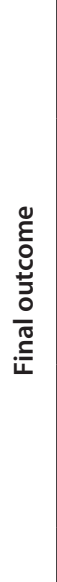 } & \multicolumn{2}{|c|}{ 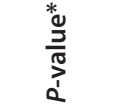 } & \begin{tabular}{l}
$\bar{\jmath}$ \\
\multirow{0}{0}{}
\end{tabular} & 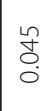 & $\left|\begin{array}{c}0 \\
\infty \\
\infty \\
0\end{array}\right|$ & $\begin{array}{l}\hat{n} \\
\text { ñ }\end{array}$ & $\begin{array}{l}\infty \\
\infty \\
\infty \\
0\end{array}$ & $\mid \begin{array}{l}\eta \\
0 \\
0\end{array}$ & ' & & $\begin{array}{l}\mathbb{8} \\
\stackrel{0}{0} \\
0\end{array}$ & & ' & 1 & , & 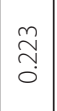 & $\begin{array}{l}\sigma \\
\tilde{O} \\
\dot{0}\end{array}$ & , & , & & $\frac{8}{\circ}$ & & $\bar{\delta}$ & \\
\hline & \multirow[t]{2}{*}{$\begin{array}{l}\frac{\Phi}{\sigma} \\
\frac{\pi}{\sigma}\end{array}$} & 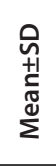 & 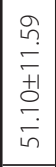 & \begin{tabular}{l}
$\stackrel{8}{0}$ \\
$\dot{\omega}$ \\
+ \\
\multirow{7}{*}{} \\
$m$ \\
$m$
\end{tabular} & 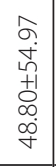 & $\begin{array}{l}\stackrel{n}{N} \\
\underset{+1}{\stackrel{n}{E}} \\
\stackrel{n}{n}\end{array}$ & 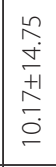 & 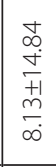 & ' & & 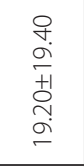 & 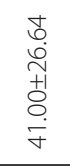 & & 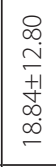 & ' & 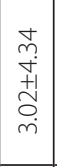 & 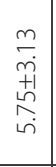 & & ' & 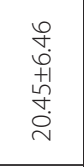 & 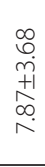 & & 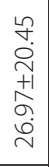 & \\
\hline & & $=$ & $\circ$ & $a$ & 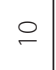 & $\sigma$ & $\circ$ & 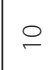 & ' & I & $\nabla$ & $\nabla$ & ' & $\stackrel{\circ}{\circ}$ & ' & $\wedge$ & $\circ$ & , & ' & 으 & 으 & ' & $\stackrel{\circ}{-}$ & \\
\hline & \multirow[t]{2}{*}{$\begin{array}{l}\text { 䒕 } \\
\text { هั }\end{array}$} & 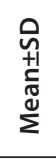 & 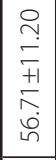 & $\begin{array}{l}8 \\
0 \\
+1 \\
0 \\
0 \\
\infty \\
\sim \\
\sim\end{array}$ & 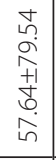 & $\begin{array}{l}\circ \\
\stackrel{0}{0} \\
\dot{9} \\
+1 \\
0 \\
0 \\
\dot{m} \\
\end{array}$ & $\begin{array}{l}2 \\
\alpha \\
\infty \\
+1 \\
\stackrel{+}{+} \\
\infty \\
\infty\end{array}$ & \begin{tabular}{|l}
$\infty$ \\
$\infty$ \\
$\infty$ \\
0 \\
0 \\
0 \\
$n$ \\
$i n$
\end{tabular} & ' & 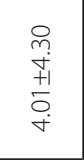 & 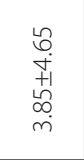 & ' & $\mid$\begin{tabular}{l}
$m$ \\
\multirow{+}{*}{} \\
+ \\
+ \\
0 \\
0 \\
$m$
\end{tabular} & 1 & 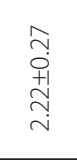 & 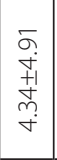 & $\begin{array}{l}\stackrel{u}{f} \\
\dot{t} \\
+ \\
m \\
m \\
m\end{array}$ & 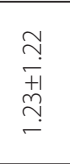 & $\begin{array}{l}\bar{m} \\
\infty \\
+1 \\
\stackrel{1}{0} \\
\stackrel{N}{N}\end{array}$ & & 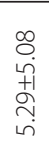 & 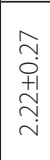 & $\begin{array}{l}\infty \\
\stackrel{n}{n} \\
+1 \\
\infty \\
\infty \\
i\end{array}$ & \\
\hline & & c & \pm & 0 & 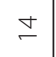 & $=$ & $\circ$ & $\circ$ & , & $\simeq$ & $a$ & , & 0 & ' & $\sim$ & $a$ & 0 & ナ & $ㅇ$ & & $\simeq$ & $\sim$ & $\underset{ }{ \pm}$ & \\
\hline \multirow{3}{*}{$\begin{array}{l}\overline{\widetilde{g}} \\
\text { 。0 }\end{array}$} & 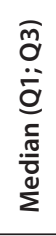 & & 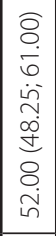 & 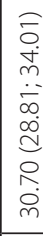 & 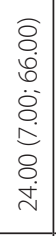 & 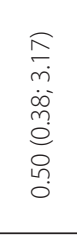 & 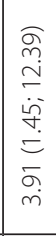 & 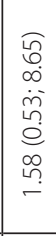 & 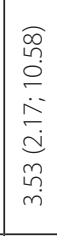 & 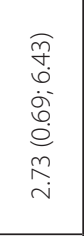 & 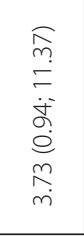 & 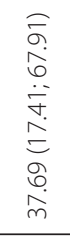 & 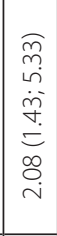 & 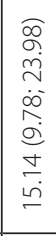 & 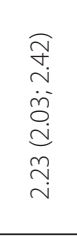 & 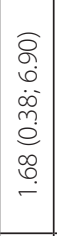 & 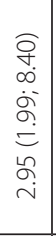 & 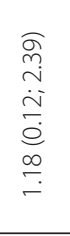 & 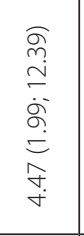 & 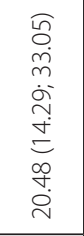 & 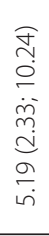 & 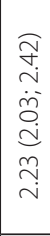 & 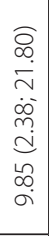 & \\
\hline & 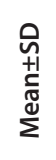 & & 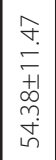 & 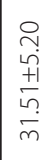 & 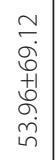 & 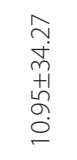 & $\begin{array}{l}\infty \\
\infty \\
\overline{\bar{\sigma}} \\
\stackrel{+}{+1} \\
\bar{\sigma} \\
\sigma\end{array}$ & 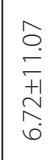 & $\begin{array}{l}\infty \\
\stackrel{\infty}{0} \\
\stackrel{0}{+1} \\
\underset{N}{+1} \\
\infty\end{array}$ & 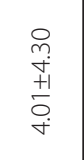 & $\begin{array}{l}\stackrel{0}{2} \\
\stackrel{i}{i} \\
+1 \\
\sim \\
\infty \\
\infty\end{array}$ & 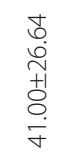 & 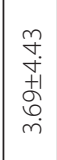 & $\begin{array}{l}\infty \\
\infty \\
\stackrel{\infty}{\sim} \\
+1 \\
\infty \\
\infty \\
\infty \\
\infty \\
-\infty\end{array}$ & $\begin{array}{l}\text { त̂ } \\
\text { +1 } \\
\text { ָ̃ } \\
\text { }\end{array}$ & 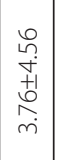 & 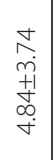 & 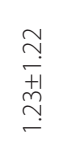 & $\begin{array}{l}\bar{m} \\
\infty \\
o \\
+1 \\
0 \\
\stackrel{0}{N}\end{array}$ & 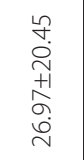 & 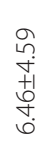 & 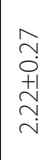 & 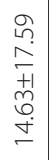 & \\
\hline & $=$ & & $\stackrel{\sim}{\sim}$ & $\stackrel{n}{\sim}$ & $\stackrel{\Delta}{\sim}$ & $\stackrel{n}{\simeq}$ & i & i & \pm & $\simeq$ & $\underline{m}$ & $\nabla$ & 0 & $\circ$ & N & $\circ$ & 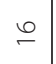 & 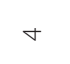 & $\stackrel{ }{0}$ & $\stackrel{\circ}{0}$ & $\approx$ & $\sim$ & $\stackrel{\sim}{\sim}$ & \\
\hline \multicolumn{3}{|c|}{ 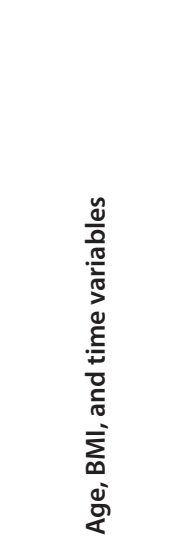 } & 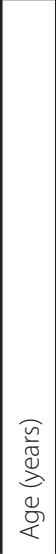 & 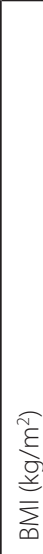 & 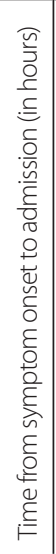 & 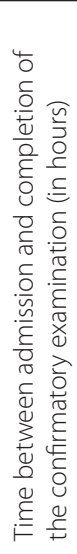 & 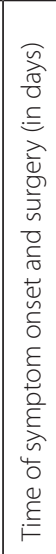 & 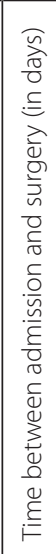 & 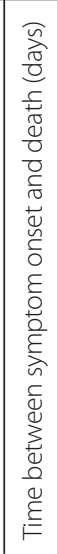 & 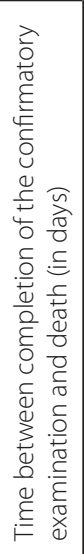 & 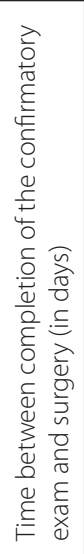 & 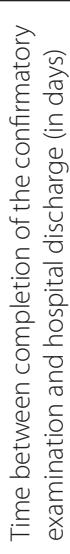 & 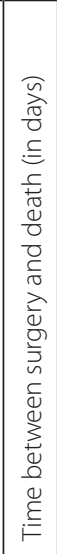 & 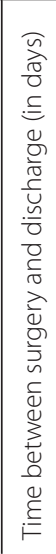 & 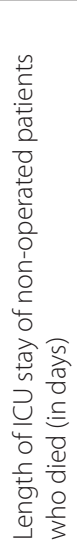 & 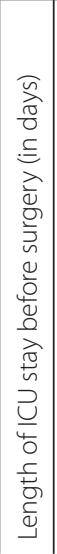 & 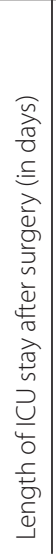 & 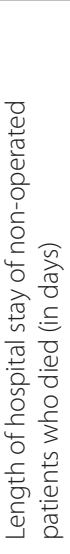 & 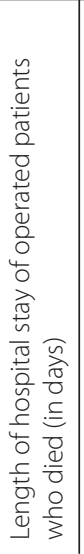 & 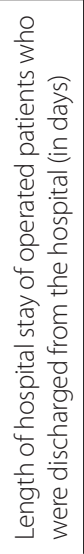 & 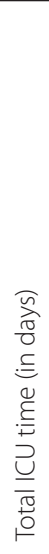 & 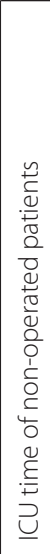 & 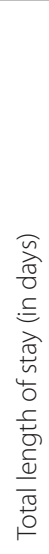 & 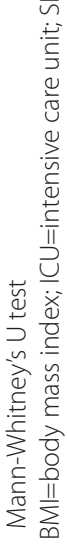 \\
\hline
\end{tabular}




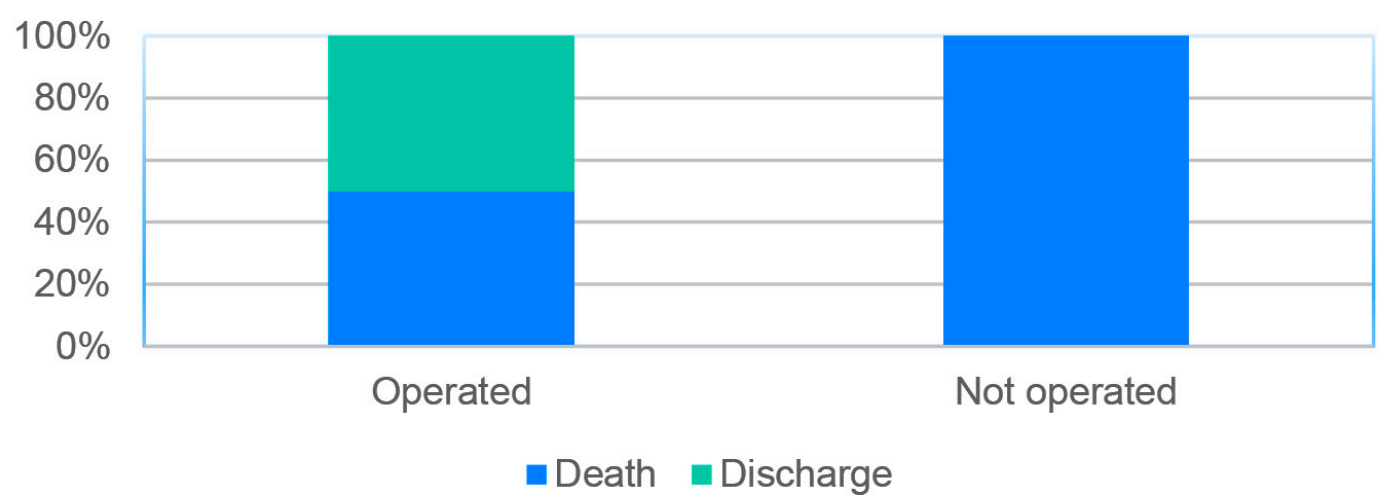

Fig. 2 - Crossover between surgical exposure and the final outcome (death or discharge). *Fisher's exact test. $P=0.114$.

surgery, 50\% survived ( $P=0.114)$. Among these non-operated patients, two decided not to undergo surgery and two died before surgery.

In the present study, no patient was positive among the variables: ethnicity, pleural effusion, Marfan syndrome or its spectrum, peripheral arterial occlusive disease, previous stroke, use of ticagrelor, and use of oral anticoagulants. No patient underwent TEE or cardiac nuclear magnetic resonance as diagnostic test for AD or even as a complementary test during the investigation.

\section{DISCUSSION}

The study obtained a small sample, despite capturing all cases of acute type A AD admitted to the service during the period, for significant analytical evaluation. Despite this low number of cases, the total number of surgeries performed per year at our service, an average of 10 cases per year, considered by many to be a high-volume referral center ${ }^{[11]}$, was similar to that of the Bristol Heart Institute, a referral hospital in cardiology in England, which published in 2017 an average of 12 cases operated per year, over a period of 17 years ${ }^{[12]}$.

The mean age found at 54.4 years is similar to that found in other studies, ranging from 43.5 to 61 years ${ }^{[7,13]}$. The male gender predominated with $79.2 \%$ of cases, ranging from 62.0 to $74.0 \%$ in the literature ${ }^{[7,13]}$. The average BMI was $31.5 \mathrm{~kg} / \mathrm{m}^{2}$, similar to other studies $^{[7,13]}$, but considering BMI $\geq 30$ as suggestive of obesity and that $60.0 \%$ of the cases in this study were in this value range, the present study demonstrated a population with an obesity profile much higher than that evidenced in the literature, around $7.5 \%[7,13]$, and this factor may have contributed to the increase in mortality. Among the symptoms, chest pain was found in $91.7 \%$ of cases and syncope in $12.5 \%$, both similar to $\operatorname{IRAD}^{[7,14]}$.

Among the complications related to dissection, the high frequency of renal failure and myocardial ischemia stands out around $45.8 \%$ and $20.8 \%$ of cases, respectively, against $9.0-35.0 \%$ and $2.5-19.2 \%$ described in the literature, respectively $[7,13,14]$. Patients were more hypertensive ( $91.7 \%$ of cases) than those registered in other services, which ranged from 58.7 to
89.0\% of cases $^{[7,13,14]}$. The most performed exam was aortic angiotomography, in $79.2 \%$ of cases, against $50.2 \%$ of the cases reported in the $\operatorname{IRAD}^{[7,14]}$. There is also a greater number of aortographies in the diagnosis (8.3\% of cases), higher than that of IRAD (about $2 \%$ of cases) ${ }^{[7,14]}$.

The average time from symptom onset to hospital admission was 53.96 hours (median=24 hours). This delay in hospital admission meant that patients were seen at a later stage ${ }^{[11]}$, which may have contributed for increased mortality, even with a response time between admission and surgery with a median of 1.58 days - less than 48 hours, as recommended, but still far above the ideal, which is $5-6$ hours ${ }^{[8,11,13]}$. The total ICU length of stay and hospitalization times were similar to those found in other studies ${ }^{[8,11,13]}$. The frequencies of reoperations and intraoperative death, both of $20.0 \%$, were higher than the average ${ }^{[8,11,13]}$, being more similar to the rates found among older patients, such as octogenarians ${ }^{[15]}$.

The lethality among the operated cases (50.0\%) was higher than the lethality found in most studies, which varies between 21.0 and $30.0 \%{ }^{[7,14]}$, but it is similar to studies carried out with patients over 75 years old, which varies between $42.0 \%$ and $83.0 \%{ }^{[15]}$. In-hospital mortality among non-operated cases was $100.0 \%$, much higher than the IRAD record ${ }^{[7]}$. It is possible that the delay in reaching a referral center has aggravated the condition of patients seen at the service, as well as the performance of more invasive imaging tests, with the use of contrast, which may also have contributed to the high rate of renal failure among patients, and the sum of these factors has been decisive for the increase in lethality; however, further studies are needed to prove or infer this increased risk.

Our service carries out the most diverse and recent surgical techniques and strategies (e.g., selective cerebral perfusion and endovascular treatment) well established in the treatment of acute type $A A D$, even though there is no significant difference between the outcomes with more aggressive strategies ${ }^{[12,16-18]}$, however, a rational strategy for the use of surgical techniques for the treatment of this condition could be implemented in the service $^{[12]}$. 
It is important that our institution, as well as all healthcare institutions, implements measures to improve the quality of the services provided, since the diagnosis of $A D$ can be very laborious in non-specialized services ${ }^{[20,21]}$. To make our institution a center specialized in the treatment of aortic diseases to which all patients from the public health system with this condition should be referred, further increasing its surgical volume and improving the final results of the treatment ${ }^{[11,20,21]}$, it is crucial the implementation of local or even national registries, such as IRAD ${ }^{[7]}$ and Nordic Consortium for Acute Type A Aortic Dissection ${ }^{[11]}$; implementation of the institution's own protocols based on the literature, establishing a routine from the initial care at the institution to rational strategies in choosing the surgical technique, as well as using the data from this work, and always improving with the use of forthcoming researches carried out at the institution ${ }^{[12,20,22]}$; and implementation of a protocol for safe surgery and improvements in health care: definition of local objectives (emergency, ICUs, wards, operating room, and all sectors involved); establishment of measures; selection, testing, and implementation of changes; and, lastly, dissemination of changes to the entire hospita [21]. Rationality, always respecting the Hippocratic principle primum non nocere, knowing that the more you move away from evidence-based practices and the care to see, feel, and listen to the patient, the closer you get to iatrogeny, thus avoiding unnecessary procedures and conduct that can delay, hinder, or even prevent the success of adequate treatment, which can culminate in the patient's death ${ }^{[23]}$.

The future of the treatment of acute type A AD is closer, with the emergence and improvement of endovascular techniques, now well established for type B cases $^{[24]}$. Although conventional open surgical treatment is still the gold standard ${ }^{[3]}$, some centers have already started treating these cases endovascularly, in situations where surgical risk is prohibitive ${ }^{[24]}$. It is expected that the development and improvement of these new techniques will reduce the cost of treating this condition, making it more widespread and accessible, in addition to bringing new perspectives for the treatment of acute type $A D^{[17]}$. The improvement and development of broader risk scores, such as the Society of Thoracic Surgeons score and the European System for Cardiac Operation Risk Evaluation score $\|^{[14,25]}$, to better contemplate the surgical risk of these patients, especially among the elderly (> 75 years), with the use other variables, such as the frailty scale, can determine a risk more compatible with reality ${ }^{[15,25]}$.

\section{Limitations}

This is a single-center, non-randomized retrospective study, with a more descriptive character and a small sample. Our service is relatively recent, in addition to having just become a referral center in the state for this condition. We have a multi-surgeon profile for aortic surgery.

\section{CONCLUSION}

The sample size was small, although our institution is considered a high-volume referral center; however, despite being a low-incidence disease, the sample clearly demonstrated the compatibility of its size with the literature. Lethality is high, and an even greater lethality was found in this study, possibly due to the delay between the symptom onset and admission to the service due to delayed diagnosis and referral of primary and secondary services, further aggravating patients, as well as due to the performance of more invasive imaging exams, using contrast, may also have contributed to the high rate of renal failure among patients. The sum of these factors has been decisive for increasing the lethality, however, more studies are needed to prove or infer a cause-effect relationship, as well as to implement actions for the early identification of these patients in non-specialized services, to reduce the time taken to referral services in the treatment of acute type A AD.

\section{No financial support. \\ No conflict of interest.}

\section{Authors' roles \& responsibilities}

PCLBB Substantial contributions to the conception or design of the work; or the acquisition, analysis, or interpretation of data for the work; drafting the work or revising it critically for important intellectual content; final approval of the version to be published

$\mathrm{RCL}$ Drafting the work or revising it critically for important intellectual content; final approval of the version to be published

PRS Drafting the work or revising it critically for important intellectual content; final approval of the version to be published

ACAM Drafting the work or revising it critically for important intellectual content; final approval of the version to be published

GMML Drafting the work or revising it critically for important intellectual content; final approval of the version to be published

AMP Drafting the work or revising it critically for important intellectual content

JVOS Substantial contributions to the acquisition, analysis, or interpretation of data for the work

CVOS Substantial contributions to the acquisition, analysis, or interpretation of data for the work

PRVOS Substantial contributions to the acquisition, analysis, or interpretation of data for the work

\section{REFERENCES}

1. Ministério da Saúde (BR). Indicadores e dados básicos [Internet]. Brasília: Ministério da Saúde; 2013 [cited 2020 Dec 7]. Available from: http:// tabnet.datasus.gov.br/cgi/idb2013/matriz.htm

2. World Health Organization - WHO. Global health estimates summary tables: projection of deaths by cause, age and sex [Internet]. Geneva: 
WHO; Jul 2013 [cited 2020 Dec 7]. Available from: https://www.who. int/healthinfo/global_burden_disease/en/

3. Erbel R, Aboyans V, Boileau C, Bossone E, Bartolomeo RD, Eggebrecht $H$, et al. 2014 ESC guidelines on the diagnosis and treatment of aortic diseases: document covering acute and chronic aortic diseases of the thoracic and abdominal aorta of the adult. The task force for the diagnosis and treatment of aortic diseases of the European society of cardiology (ESC). Eur Heart J. 2014;35(41):2873-926. Erratum in: Eur Heart J. 2015;36(41):2779. doi:10.1093/eurheartj/ehu281.

4. Mokashi SA, Svensson LG. Guidelines for the management of thoracic aortic disease in 2017. Gen Thorac Cardiovasc Surg. 2019;67(1):59-65. doi:10.1007/s11748-017-0831-8.

5. Elsayed RS, Cohen RG, Fleischman F, Bowdish ME. Acute type A aortic dissection. Cardiol Clin. 2017;35(3):331-45. doi:10.1016/j.ccl.2017.03.004.

6. Salameh MJ, Ratchford EV. Aortic dissection. Vasc Med. 2016;21(3):27680. doi:10.1177/1358863X16632898.

7. Hagan PG, Nienaber CA, Isselbacher EM, Bruckman D, Karavite DJ, Russman $\mathrm{PL}$, et al. The international registry of acute aortic dissection (IRAD): new insights into an old disease. JAMA. 2000;283(7):897-903. doi:10.1001/jama.283.7.897.

8. Olsson C, Thelin S, Ståhle E, Ekbom A, Granath F. Thoracic aortic aneurysm and dissection: increasing prevalence and improved outcomes reported in a nationwide population-based study of more than 14,000 cases from 1987 to 2002. Circulation. 2006;114(24):2611-8. doi:10.1161/ CIRCULATIONAHA.106.630400.

9. Mauban EC, Pyeritz R, Hutchison S, Ota T, Upchurch G, Nour K, et al. Diagnostic imaging for acute aortic dissection: imaging sensitivity and preference revisited. J Am Coll Cardiol. 2018;71(11_Suppl):A2073.

10. Bonow RO, Mann DL, Zipes DP, Libby P, Bomow RO, Braunwald E editors. Braunwald's Heart Disease: A textbook of cardiolvascular medicine. 10 ed. Philadelphia (PA); Saunders; 2012. 2136 p.

11. Chikwe J, Cavallaro P, Itagaki S, Seigerman M, Diluozzo G, Adams DH. National outcomes in acute aortic dissection: influence of surgeon and institutional volume on operative mortality. Ann Thorac Surg. 2013;95(5):1563-9. doi:10.1016/j.athoracsur.2013.02.039.

12. Bashir M, Shaw M, Field M, Kuduvalli M, Harrington D, Fok M, et al. Repair of type A dissection-benefits of dissection rota. Ann Cardiothorac Surg. 2016;5(3):209-15. doi:10.21037/acs.2016.05.09.

13. Chiappini B, Schepens M, Tan E, Dell' Amore A, Morshuis W, Dossche K, et al. Early and late outcomes of acute type A aortic dissection: analysis of risk factors in 487 consecutive patients. Eur Heart J. 2005;26(2):180-6. doi:10.1093/eurheartj/ehi024.

14. Evangelista A, Maldonado G, Gruosso D, Teixido G, Rodríguez-Palomares
J, Eagle K. Insights from the international registry of acute aortic dissection. Glob Cardiol Sci Pract. 2016;2016(1):e201608. doi:10.21542/ gcsp.2016.8.

15. Santini F, Montalbano G, Messina A, D'Onofrio A, Casali G, Viscardi $F$, et al. Survival and quality of life after repair of acute type A aortic dissection in patients aged 75 years and older justify intervention. Eur J Cardiothorac Surg. 2006;29(3):386-91. doi:10.1016/j.ejcts.2005.12.016.

16. Narayan P, Rogers CA, Benedetto U, Caputo M, Angelini GD, Bryan AJ. Malperfusion rather than merely timing of operative repair determines early and late outcome in type A aortic dissection. JThorac Cardiovasc Surg. 2017;154(1):81-6. doi:10.1016/j.jtcvs.2017.03.041.

17. De Santo LS. Acute aortic dissection: the conundrum of severity of disease, extent of surgery and end-organ function. JThorac Cardiovasc Surg. 2018;155(6):2275-6. doi:10.1016/j.jtcvs.2018.01.063.

18. Tsagakis K, Pacini D, Di Bartolomeo R, Gorlitzer M, Weiss G, Grabenwoger $M$, et al. Multicenter early experience with extended aortic repair in acute aortic dissection: is simultaneous descending stent grafting justified? J Thorac Cardiovasc Surg. 2010;140(6 Suppl):S1 16-20; discussion S142-6. doi:10.1016/j.jtcvs.2010.07.066.

19. Bonser RS, Ranasinghe AM, Loubani M, Evans JD, Thalji NM, Bachet JE, et al. Evidence, lack of evidence, controversy, and debate in the provision and performance of the surgery of acute type A aortic dissection. J Am Coll Cardiol. 2011;58(24):2455-74. doi:10.1016/j.jacc.2011.06.067.

20. Lawton JS, Liu J, Kulshrestha K, Moon MR, Damiano RJ Jr, Maniar H, et al. The impact of surgical strategy on survival after repair of type A aortic dissection. J Thorac Cardiovasc Surg. 2015;150(2):294-301.e1. doi:10.1016/j.jtcvs.2015.03.023.

21. Institute for Healthcare Improvement. Improving Health and Health Care Worldwide [Internet]. Boston (MA): Institute for Healthcare Improvement; c2020 [cited 2020 Dec 7]. Available from: http://www.ihi.org

22. Cohen RG, Hackmann AE, Fleischman F, Baker CJ, Cunningham MJ, Starnes VA, et al. Type A aortic dissection repair: how i teach it. Ann Thorac Surg. 2017;103(1):14-7. doi:10.1016/j.athoracsur.2016.10.048.

23. Institute of Medicine (US) Committee on Quality of Health Care in America. To err is human: building a safer health system. Kohn LT, Corrigan JM, Donaldson MS, editors. Washington (DC): National Academies Press (US); 2000.

24. Shah A, Khoynezhad A. Thoracic endovascular repair for acute type A aortic dissection: operative technique. Ann Cardiothorac Surg. 2016;5(4):389-96. doi:10.21037/acs.2016.07.08.

25. Gomibuchi T, Seto T, Komatsu M, Tanaka H, Ichimura H, Yamamoto T, et al. Impact of frailty on outcomes in acute type A aortic dissection. Ann Thorac Surg. 2018;106(5):1349-55. doi:10.1016/j.athoracsur.2018.06.055. 